\title{
Interdisciplinary care for spinal cord injured refugees
}

\author{
Margaret W. Jones ${ }^{1} \cdot$ Deborah A. Crane $^{1}$
}

Received: 19 June 2019 / Revised: 11 March 2020 / Accepted: 11 March 2020

(c) International Spinal Cord Society 2020

\begin{abstract}
Introduction For war-injured refugees, spinal cord injury (SCI) is a leading cause of catastrophic neurologic injury and literature focused on the care of this vulnerable population is sparse. This case series describes the unique challenges presented to an outpatient interdisciplinary rehabilitation team in providing SCI care in the USA for refugees who suffered their SCI in their home countries.

Case presentation Our interdisciplinary rehabilitation team faced challenges related to language, cultural and educational barriers which impacted the typical standard rehabilitation care offered to these individuals. Many of the individuals were focused on curative treatments. As such, managing expectations and educating individuals to rehabilitation practices for chronic SCI and lack of curative treatments for associated medical complications affected the provision of care across all disciplines of the rehabilitation team.

Discussion This case series showcases the challenges of caring for international refugees with SCI. The care offered to these individuals highlights the benefits that an interdisciplinary SCI rehabilitation approach can provide to comprehensively care for this vulnerable population.
\end{abstract}

\section{Introduction}

There is a paucity of literature on the delivery of medical and rehabilitative care for refugees with spinal cord injury (SCI) who have resettled in the United States. The limited body of literature includes descriptive accounts of the experiences of primary care providers $[1,2]$. There is data on the prevalence of significant neurologic injuries experienced by refugees in their home countries, including SCI, traumatic brain injury, peripheral nerve injury, and other trauma [3]. We present a case series highlighting the interdisciplinary approach employed in caring for six refugees with SCI who established care in a multidisciplinary outpatient Rehabilitation Medicine clinic.

The standard interdisciplinary rehabilitation approach offered to this cohort had to be tailored to meet their unique

These authors contributed equally: Margaret W. Jones, Deborah

A. Crane

Deborah A. Crane

dacrane@uw.edu

1 Department of Rehabilitation Medicine, University of Washington, Seattle, WA, USA needs. The rehabilitation team's main goals were to help these men acquire basic education about their injuries, to establish appropriate medical care and to prevent secondary medical complications. Furthermore, the provision of rehabilitation care was affected by limited resources for these individuals including limited benefits for therapies and equipment.

\section{Case series}

\section{Case 1}

A 42-year-old Somali man with T9 AIS A paraplegia from a gunshot wound presented 7 years after his SCI. He had had no formal schooling, but had taught himself to read and write Somali. He used a Foley catheter for bladder management, but had no bowel program and had fecal incontinence. He had an inadequate manual wheelchair and was dependent on his wife for transfers and basic self-care tasks. He also had poorly controlled neuropathic pain. He was admitted to inpatient rehabilitation for 12 days. Upon discharge, he had improved bowel and bladder management, had obtained appropriate equipment, and was more independent with mobility and self-care. However, he continued to decline neuropathic pain management due to not wanting 
to take medications that were not curative. In outpatient follow up, he continued to perseverate on needing advanced imaging to guide curative surgery for his SCI.

\section{Case 2}

A 35-year-old Somali man with S2 AIS A SCI with cauda equina syndrome from a gunshot wound presented 8 years after his injury. He was followed in International Medicine clinic, a specialized primary care clinic for individuals from international backgrounds, for 5 years before referral to Rehabilitation Medicine Clinic, where he was eventually referred for assistance in reducing urinary tract infection (UTI) frequency. His bowel and bladder management included a colostomy and an intermittent catheterization program. He had moderately controlled neuropathic pain.

\section{Case 3}

A 58-year-old Somali man with L2 (L3 motor) AIS A SCI from a gunshot wound presented 20 years following his SCI. He suffered with bowel and bladder incontinence, poorly controlled neuropathic pain, and untreated hypertension. He was ambulatory with crutches, but persistently requested a power wheelchair and was fixated on surgical fixation of his SCI. Despite attempting various strategies and education, he had great difficulty with medication adherence and ultimately developed chronic kidney disease with impending hemodialysis requirement.

\section{Case 4}

A 22-year-old Somali man with T9 AIS C paraplegia from a gunshot wound presented 6 years after he sustained his SCI. He used a Foley catheter for bladder management, had no bowel program and was stooling every third day, had a history of multiple wounds, and struggled with poorly controlled pain, extensive joint contractures, and inadequate equipment. He received a manual wheelchair for mobility but felt he would be best be served by a power wheelchair. After extensive bowel and bladder training he was able to independently perform intermittent catheterization after years of indwelling catheter use.

\section{Case 5}

A 28-year-old Somali man with T12 AIS B paraplegia from a gunshot wound presented 3 years after his SCI. He was unable to read or write Somali or English. He managed his bladder with a Foley catheter, but had frequent UTIs. For bowel management, he strained and required occasional manual evacuation. He possessed inadequate adaptive equipment and had a sacral wound. He was also very focused on curative treatment. He participated in outpatient therapies and learned to independently and safely perform transfers and use a manual wheelchair for home and community mobility. He refused intermittent catheterization until meeting with urology to discuss curative treatment for his bladder.

\section{Case 6}

A 44-year-old Iraqi man with cauda equina syndrome presented 11-12 years after occurrence of his SCI. He sustained his injuries as a result of years of torture and incarceration, consequently, the exact timing of his injuries was not clear. In addition to his SCI, he also suffered multiple head injuries, tooth extractions, and fractured limbs. His youngest child died in a refugee camp during his immigration to the United States. Upon presentation in Rehabilitation Medicine clinic, he was dealing with inaccessible housing, chronic neuropathic and axial back pain, bowel and bladder dysfunction, spinal instability, and memory deficits. He was ambulatory for short distances with a cane, but was limited primarily due to pain. He requested a power wheelchair, which was challenging to obtain due to architectural accessibility issues as well as insurance funding. He was already connected with psychiatric services through referral from the International Medicine clinic.

\section{Discussion}

There are numerous challenges that the rehabilitation team faced in providing care for this cohort of refugees with SCI. All team members battled language, cultural, and educational barriers. Even with the existence of a strong institutional interpreter system, team members struggled with needing longer encounters to account for interpretation time and at times, having to rely on telephone interpreters, which can make hands-on bowel and bladder education and training more difficult. In the authors' experiences, individuals from developing nations often have a poor understanding of the role of rehabilitation. Thus it was difficult for team members to explain their roles as well as goals of interventions that were provided. Many of the men in this cohort had very limited educational backgrounds; this made providing SCI education and written educational materials difficult.

Challenges more specific to the medical providers included pharmacological management, bowel and bladder management, and managing patient expectations. Frequently, individuals seemed to struggle with medication adherence. The clinic is partnered with an in-house pharmacy that can package medications using systems to bypass 
lack of English or native language literacy. However, individuals still seemed to misunderstand the need to take most medications chronically even with symptomatic improvement. It was not uncommon for individuals in this cohort to discontinue neuropathic pain medications once their neuropathic pain subsided only to experience a recurrence of pain. Similar issues arose with medications to reduce urinary urgency and incontinence. The men in this cohort frequently had unrealistic expectations about what curative treatments for their injuries existed. They were often insistent about receiving referrals to surgical specialists for curative surgeries and undergoing advanced diagnostics to guide curative treatments. There was often disinterest in learning more about compensatory management strategies, such as establishing a routine bowel program or learning to perform self-catheterization and demand for medications or other treatments that would resolve the $\mathrm{SCI}$ and related issues.

Other members of the interdisciplinary team faced barriers in the delivery of care for this population as well. For physical and occupational therapists, procuring adaptive equipment was difficult due to funding limitations for individuals newly settled in the USA This was confounded by some individuals' expectations for power mobility when the standard of care in the USA for their impairment was a manual wheelchair or even assistive gait aid. Specific difficulties that nurses faced were trying to overcome individuals' limited educational backgrounds and difficulties with written educational materials related to language barriers and illiteracy. Social work was limited in translating available resources to newly resettled refugees and sometimes assumed case management roles, outside of the typical scope of practice in our clinic. Psychology services may be underutilized in this population due to individuals' preference to prioritize medical services over addressing mood.

Based on our team's experience working with this patient population, we have identified some areas for future growth in the care of this or similarly challenging populations. The ability to offer culturally-specific peer support for refugees with traumatic and catastrophic neurologic injury may help individuals in their adjustment to disability and in identifying specific goals for working on their functional impairments. Another area that would benefit from further attention is the addition of comprehensive case management for these individuals to help navigate the healthcare system. The home institution for these cases is fortunate to have cultural liaisons to help with some of these dynamics but this role is not that of a formal case manager. Finally, these cases provided a reminder of the ongoing need to educate providers in other specialties of the role that Rehabilitation Medicine can play in helping to care for newly settled refugee.

\section{Conclusion}

There are myriad challenges that arose in assuming the care of refugees with SCI who resettled in the USA, the greatest of which included language and cultural barriers, financial limitations and managing expectations for curative treatments. Through proactive communication and interdisciplinary care, we succeeded in helping these individuals achieve improved functional mobility, acquire appropriate adaptive equipment, and gain greater independence with self-care including improved bowel and bladder management.

\section{Compliance with ethical standards}

Conflict of interest The authors declare that they have no conflict of interest.

Publisher's note Springer Nature remains neutral with regard to jurisdictional claims in published maps and institutional affiliations.

\section{References}

1. Moreno A, Grodin MA. Torture and its neurological sequelae. Spinal Cord. 2002;40:213-23.

2. Langerkvist B. Rehabilitation research under fire. Scand J Soc Med. 1998;26:85-6.

3. Hermansoon AC, Thyberg M, Timpka T. War-wounded refugees: the types of injury and influence of disability on well-being and social integration. Med Confl Surviv. 1996;12:284-302. 